\title{
ON MULTIPLY TRANSITIVE GROUPS I
}

\author{
HIROSI NAGAO \\ Dedicated to the memory of Professor Tadasi Nakayama
}

The purpose of this paper is to prove the following three theorems which were announced in [2].

Theorem 1. Let $G$ be a quadruply transitive group on $\{1,2, \ldots, n\}$ and $H$ the subgroup of $G$ consisting of all the elements leaving the two letters 1 and 2 invariant. If $G$ is of even degree and $H$ contains a normal subgroup $Q$ which is regular on $\{3,4, \ldots, n\}$, then $G$ is one of the following groups: $S_{4}, S_{6}$ or A6.

TheOREM 2. Let $G$ be a quintuply transitive group on $\{1,2, \ldots, n\}$ and $H$ the subgroup of $G$ consisting of all the elements leaving the three letters 1,2 and 3 invariant. If $H$ contains a normal subgroup $Q$ which is regular on $\{4$, $5, \ldots, n\}$, then $G$ is one of the following groups: $S_{5}, S_{6}, S_{7}, A_{7}$ or $M_{12}$.

The following theorem is an improvement of a theorem of Wielandt ([4], Satz 1).

THEOREM 3. Let $G$ be a k-fold transitive group of degree $n$. If the outer automorphism group of any simple subgroup of $G$ is solvable, then $k \leq 6$ unless $G$ is $S_{n}$ or $A_{n}$.

We use standard notations throughout. For a set $X$ let $|X|$ denote the number of elements of $X$. For a subset $X$ of a group $G$ let $N_{G}(X)$ denote the normalizer of $X$ in $G$, and the centralizer of $X$ in $G$ is denoted by $C_{G}(X)$.

\section{Proof of Theorem 1}

We first prove the following lemma which will be used in this and the next sections.

Received January 18, 1965. 
LemmA ${ }^{11}$. Let $V$ be a vector space over a field and $\rho$ a nilpotent linear transformation of $V$. If $\rho^{n}=0$ then

$$
\operatorname{dim} V \leq \boldsymbol{n} \operatorname{dim} V_{0}
$$

where $V_{0}=\{v \in V ; \rho v=0\}$.

Proof. We prove the lemma by the induction on $n$. For $n=1$, the lemma is trivial. Let $W=\rho V$. Then $W \simeq V / V_{0}$. Since $\rho^{n-1} W=0$ we have, by the hypothesis of induction,

$$
\operatorname{dim} W \leq(n-1) \operatorname{dim} W_{0},
$$

where $W_{0}=W \cap V_{0}$. Therefore we have

$$
\begin{aligned}
\operatorname{dim} V & =\operatorname{dim} W+\operatorname{dim} V_{0} \\
& \leq(n-1) \operatorname{dim} W_{0}+\operatorname{dim} V_{0} \\
& \leq \boldsymbol{n} \operatorname{dim} V_{0} .
\end{aligned}
$$

Proof of Theorem 1 . Since $Q$ is regular on $\{3,4, \ldots, n\}$ and $n$ is even, $Q$ is of even order. Now $Q$ is a regular normal subgroup of $H$ which is doubly transitive on $\{3,4, \ldots, n\}$, therefore $Q$ is an elementary abelian subgroup of exponent 2 ([3], 11.3, (a)) and the unique minimal normal subgroup of $H$ ([3], $11.4,11.5)$.

Let $s \neq 1$ be an element of $Q$. We may assume

$$
s=(1): 2,(3,4) \cdots
$$

Since $G$ is quadruply tranistive there is an element $x$ in $G$ such that

$$
x=\left(\begin{array}{lllll}
1 & 2 & 3 & 4 & \cdots \\
3 & 4 & 1 & 2 & \cdots
\end{array}\right)
$$

Let $t=x^{-1} s x$. Then

$$
t=(1,2)(3)(4) \cdots
$$

and $t$ fixes only two letters 3 and 4 . Since $t$ is in $N_{G}(H)$ and $Q$ is the unique minimal normal subgroup of $H, t^{-1} Q t=Q$ and $t$ induces an automorphism $\tau$ of $Q$. Let $Q_{0}$ be the subgroup of $Q$ consisting of all the elements left invariant by $\tau$. From the regularity of $Q, s$ is in $Q_{0}$. Let

1) The lemma of this general form is due to the suggestion by Professor N. Ito. The lemma was first stated in more special form. 


$$
r=(1)(2)(3, \alpha) \cdots
$$

be an element in $Q$ which is different from $s$, then $\alpha \neq 1,2,3,4$. If $\alpha \rightarrow \alpha^{\prime}$ under $t$ then $\alpha^{\prime} \neq \alpha$ and

$$
r^{\tau}=t^{-1} r t=(1)(2)\left(3, \alpha^{\prime}\right) \cdots
$$

is different from $r$. Thus we have $Q_{0}=\{1, s\}$ and $\left|Q_{0}\right|=2$. Applying Lemma for $\rho=\tau-1$, we have $|Q| \leq 4$, therefore $|Q|=n-2=2$ or $4, n=4$ or 6 . The quadruply transitive group of degree 4 or 6 is clearly $S_{4}, A_{6}$ or $S_{6}$.

\section{Proof of Theorem 2}

In the same way as Theorem 1 we have first the following proposition.

Proposition. Let $G$ be a quintuply transitive group on $\{1,2, \ldots, n\}$ and $H$ the subgroup of $G$ consisting of all the elements leaving the three letters 1,2 and 3 invariant. If $n$ is divisible by 3 and $H$ contains a normal subgroup $Q$ which is regular on $\{4,5, \ldots, n\}$, then $G$ is $S_{6}$ or $M_{12}$.

Proof. Since $H$ is doubly transitive on $\{4,5, \ldots, n\}$, where $n$ is a multiple of 3 , and $Q$ is a regular normal subgroup of $H, Q$ is an elementary abelian subgroup of exponent 3 and the unique minimal normal subgroup of $H$.

Let $s \neq 1$ be an element of $Q$. We may assume

$$
s=(1)(2)(3)(4,5,6) \cdots
$$

Since $G$ is quintuply transitive there is an element $x$ in $G$ such that

$$
x=\left(\begin{array}{llllll}
1 & 2 & 4 & 5 & 6 & \cdots \\
4 & 5 & 1 & 2 & 3 & \cdots
\end{array}\right) .
$$

Let $t=x^{-1} s x$. If $3 \rightarrow \alpha$ under $x$ then

$$
t=(1,2,3)(4)(5)(\alpha) \cdots
$$

and $t$ fixes only three letters $4,5, \alpha$. Since $t^{-1} H t=H, t$ induces an automorphism $\tau: x \rightarrow t^{-1} x t$ of $Q$, whose order is 3. Let $Q_{0}$ be the subgroup of $Q$ consisting of all the elements left invariant by $\tau$. Since $Q$ is regular on $\{4,5, \ldots$, $n\}$ and both $s$ and $s^{\tau}=t^{-1} s t$ take 4 to 5 , we have $s=s^{\tau}, s \in Q_{0}$ and $t$ fixes 6 . Therefore $\alpha=6$. Let

$$
r=(1)(2)(3)(4, \beta, \gamma) \cdots
$$


be an element in $Q$ which is different from $s$ and $s^{2}$, then $\beta \neq 1,2,3,4,5,6$. If $\beta \rightarrow \beta^{\prime}$ under $t$, then $\beta \neq \beta^{\prime}$ and

$$
r^{\tau}=t^{-1} r t=(1)(2)(3)\left(4, \beta^{\prime}, \gamma^{\prime}\right) \cdots
$$

is different from $r$. Thus we have $Q_{0}=\left\{1, s, s^{2}\right\}$ and $\left|Q_{0}\right|=3$. Applying Lemma for $\rho=\tau-1$, we have $|Q| \leq\left|Q_{0}\right|^{3}=27$, since $(\tau-1)^{3}=0$. Therefore $|Q|=n-3=3,9$ or $27, n=6,12$ or 30 . If $n=6, G$ must be $S_{6}$. It is known that a quadruply transitive group of degree 11 is $S_{11}, A_{11}$ or $M_{11}$ ([1], p. 77). Therefore if $n=12, G$ is one of the groups $S_{12}, A_{12}$ or $M_{12}$. But among these groups only $M_{12}$ satisfies the assumption. If $n=30$, then $n=2 \cdot 13+4$ and by a theorem of Miller ([1], Theorem 5.7.2) $G$ must be $S_{30}$ or $A_{30}$. But in both cases $G$ does not satisfy the assumption.

Proof of Theorem 2. Since $H$ is doubly transitive on $\{4,5, \ldots, n\}, \boldsymbol{Q}$ is an elementary abelian subgroup. Let $V$ be the subgroup consisting of all the elements leaving the five letters $1,2,3,4$ and 5 invariant, and let $\Delta=\{1,2,3$, $4,5, \cdots\}$ be the set of all letters left invariant by $V$. By a theorem of Witt [5] $N=N_{G}(V)$ is quintuply transitive on $\Delta$. Let $N^{\Delta}$ be the restriction of $N$ on 4. Then the kernel of the natural homomorphism $\varphi: N \rightarrow N^{\Delta}$ is $V$ and we have $N / V \simeq N^{\Delta}$. The permutation group $N^{\Delta}$ on $\Delta$ is a quintuply transitive group such that only the identity leaves five letters invariant. By a theorem of Jordan ([1], p. 72) $N^{\Delta}$ is one of the following groups: $S_{5}, S_{6}, A_{7}$ or $M_{12}$. Therefore $|\Delta|=5,6,7$ or 12 .

Let $H_{0}=H \cap N$. Then $H_{0}^{\Delta}=\varphi\left(H_{0}\right)$ is the subgroup of $N^{\Delta}$ consisting of all the elements leaving the three letters 1,2 and 3 invariant. Let $Q_{0}=Q \cap N$. Since $Q$ is regular on $\{4,5, \ldots, n\}$, there is an element $s$ in $Q$ such that

$$
s=(1)(2)(3)(4,5, \ldots) \cdots
$$

and then, by the regularity of $Q, s \in C_{G}(V), s \in Q_{0}$. Thus $Q_{0} \neq 1$. $Q_{0}$ is isomorphic to $Q_{0}^{\Delta}=\varphi\left(Q_{0}\right)$ and $Q_{0}^{\Delta}$ is a normal subgroup of a doubly transitive group $H_{0}^{\Delta}$ on $\Delta-\{1,2,3\}$. Therefore $Q_{0}^{\Delta}$ is transitive on $\Delta-\{1,2,3\}$ and hence regular on it. Thus we have $\left|Q_{0}^{\Delta}\right|=\left|Q_{0}\right|=|\Delta|-3=2,3,4$ or 9 . Since $Q_{0}$ is a subgroup of the elementary abelian group $Q$, the exponent of $Q$ must be 2 or 3 . If the exponent is 2 , by Theorem $1, G$ is a transitive extension of $S_{4}{ }^{\circ}, S_{6}$ or $A_{6}$, therefore $G$ must be one of the groups $S_{5}, S_{7}$ or $A_{7}$. If the exponent is 3 , by Proposition, $G$ is $S_{6}$ or $M_{12}$. 


\section{Proof of Theorem 3}

Let $X$ be a 7 -fold transitive group on $\{1,2, \ldots, n\}$, which is different from $S_{n}$ and $A_{n}, G$ the subgroup of $X$ consisting of all the elements leaving the two letters 1 and 2 invariant, and let $H$ be the subgroup consisting of all the elements leaving the five letters $1,2,3,4$ and 5 invariant. The group $G$ is quintuply transitive on $\{3,4, \ldots, n\}$. By Hilfssatz (2) in [4], $H$ contains a normal subgroup which is regular on $\{6,7, \ldots, n\}$. Therefore, by Theorem $2, G$ is one of the following groups: $S_{5}, S_{6}, S_{7}, A_{7}$ or $M_{12}$. Since $M_{12}$ has no transitive extension, $G$ is a symmetric or alternating group and hence $X$ is $S_{n}$ or $A_{n}$. This is a contradiction.

\section{REFERENCES}

[1] M. Hall, The Theory of Groups, Macmillan, New York (1959).

[2] H. Nagao, On a theorem of Wielandt, Proc. Japan Acad., 40, 793-794 (1964).

[3] H. Wielandt, Vorlesungen über Permutationsgruppen, Tübingen (1955).

[4] H. Wielandt, Über den Transitivitätsgrad von Permutationsgruppen, Math. Z. 74, 297298 (1960).

[5] E. Witt, Die 5-fach transitiven Gruppen von Mathieu, Abh. Math. Sem. Univ. Hamburg, 12, 256-264 (1937).

\section{Osaka City University}

